\title{
Moving forward with ethnomethodological approaches to analysing mathematics classroom interactions
}

\author{
Jenni Ingram ${ }^{1}$ D \\ Accepted: 30 May 2018 / Published online: 9 June 2018 \\ (c) The Author(s) 2018
}

\begin{abstract}
Ethnomethodological approaches to research such as Interactionism, Conversation Analysis and Discursive Psychology have so far played only a small part in mathematics education research. This is in contrast to the wider use of these approaches in language education, medical discourse research, and legal discourse research. This article outlines the key principles an ethnomethodological approach follows, the methods used and the necessary reconceptualisation of some key concepts that are the focus of much mathematics education research. These are illustrated with some key examples from research in mathematics education, before looking at other fields for potential opportunities not yet realised within research in mathematics classrooms. These opportunities include a range of research questions already receiving a great deal of attention within the field but are yet to be examined from an ethnomethodological perspective. They also include new approaches that involve combining different research approaches which have been used to great effect in other fields.
\end{abstract}

Keywords Ethnomethodology $\cdot$ Conversation analysis $\cdot$ Discursive psychology $\cdot$ Interactionism

\section{Introduction}

The study of language use and classroom interaction within mathematics education have been approached in quite diverse ways, as is reflected in the recent contributions to the language groups within the Congress of European Research in Mathematics Education and the International Congress on Mathematical Education. Researchers are now drawing upon a range of theoretical approaches from a range of disciplines such as linguistics, sociology and anthropology, and combining these with theories from mathematics education that are enabling us to explore the complex relationship between language and mathematics learning. As Barwell argues, however, there is a need in mathematics education research for "a more thorough approach to the analysis of classroom interaction" (2003, p. 206) and ethnomethodological approaches offer this thoroughness.

In this article, I consider the theoretical and methodological approaches to the analysis of interaction that originate in Garfinkel's (1967) ethnomethodology that have been used

Jenni Ingram

Jenni.Ingram@education.ox.ac.uk

1 University of Oxford, Oxford, UK in mathematics education research, including Conversation Analysis (Forrester and Pike 1998; Ingram and Elliott 2016), Interactionism (e.g. Krummheuer 2007), and Discursive Psychology (e.g. Barwell 2002). Ethnomethodological approaches share a primary interest in social actions, how language is used to achieve different things, rather than in language itself. It is both a theoretical perspective on social actions and a method for researching these actions. The unit of analysis is interaction rather than the individual, with the focus on what participants are doing with their utterances or gestures in this interaction. For example, Forrester and Pike (1998) studied what it means to estimate through analysing the different activities teachers and students take part in when estimating, rather than trying to uncover what a student does or does not understand by estimation. This focus on action is underpinned by the idea that by saying things in different ways, different things are achieved. These different ways not only include our choice of words but also how we say things. When we act, we do so in ways that show our understanding of the interactional context in which we are acting, but our actions are also reflexively constructing this social context or situation. Through studying these actions within the context that they occur, we can understand how classrooms are organised, and how particular mathematical practices or activities are accomplished. Importantly, 
within these approaches, contexts are co-constructed by teachers and students and are thus fluid and dynamic. These approaches are therefore particularly powerful when we want to examine the co-construction of mathematical knowledge and practices, such as explaining, arguing or convincing, but can also be used to explore identity work and the construction of knowledge and expertise. As Bruner (1990) pointed out there is a focus in much research about what people say, rather than on what they actually do, stating that it is "curious that there are so few studies that go in the other direction: How does what one does reveal what one thinks or feels or believes?" (p. 17). Ethnomethodological approaches, and Discursive Psychology in particular, seek to address this other direction.

Radford (2008) suggests we focus on three aspects of theory when we intend to use them in our research: theoretical principles; methodology; and research questions, and the first part of this article is structured around these aspects. There is a risk when combining theories from different disciplines of conflicting theoretical assumptions, particularly when concepts or frameworks are used without the theoretical underpinnings that give these meanings (Moschkovich 2016). I begin this article by outlining the origins of ethnomethodology and the fundamental principles that underpin any ethnomethodological approach. Ethnomethodological approaches are made visible through the enactment of these underlying principles in their methodology, which contrasts with many current approaches to the analysis of interactions in the teaching and learning of mathematics. These theoretical and methodological differences are illustrated through recent examples of research within mathematics education, thus exemplifying the diverse range of research questions that can be addressed through such an approach. This article then ends by exploring how these ethnomethodological approaches have moved forward in other disciplines, such as social psychology, medicine and linguistics, and the potential these developments may have for mathematics education research. Whilst ethnomethodological approaches can be used to study any social actions, they have largely been used to study talk-in-interaction, hence their relevance to the field of language use within mathematics education.

\section{Origins and principles of ethnomethodology}

Ethnomethodology was developed by Garfinkel (1967) and arose out of a dissatisfaction with the then current approaches within the social sciences where researchers constructed theories or models, which were then applied in the analysis of interaction. Garfinkel argued that these displayed the researcher's understanding of the situation and not necessarily that of the individuals involved in the interaction itself. Ethnomethodology shifts the focus of analysis to the participants' understanding, which they reveal through their actions, such as what they say and how they say it. Interpretations offered by researchers cannot be considered significant or important unless the participants orient to, recognise or use these interpretations themselves. The term ethnomethodology specifically refers to "the study of common-sense knowledge and the methods we use to make sense of and act upon" the situation in which we find ourselves (Heritage 1984, p. 4). These methods or procedures are not necessarily explicit, and teachers' or students' actions are not necessarily conscious, but they reveal how teachers and students are making sense of the interaction. The goal is to describe the methods and assumptions we use in our interactions to make sense of the interaction and that enable us to achieve what we want to achieve. Many other approaches to the analysis of classroom interactions cannot sufficiently explain this sense-making because they do not take into account how teachers or students themselves make sense of the interaction.

There are three fundamental principles that underpin any ethnomethodologically based study which are evident in both the ways in which a study is designed, including how data is collected, and in how the data is analysed. These principles are that all social interactions are ordered, all interactions are context-bound (indexicality), and that all details of an interaction need to be considered in any analysis, nothing can be decided 'a priori' to be irrelevant. It is these principles that mark the key differences between an ethnomethodological approach and other studies of language in the mathematics classroom.

\subsection{Social interactions are orderly}

A key driving assumption made within ethnomethodology is that all social interactions are orderly, and this order is coconstructed by participants in social interaction. This idea of order is also referred to as norms (Yackel and Cobb 1996), rules (Mchoul 1978), routines and rituals (Krummheuer 2011), or cultural codes (Bruner 1996). Fundamentally within an ethnomethodological approach these norms are not something that participants are obliged to follow, rather they are co-constructed by participants and are used by them as a structure to construct their own actions and to interpret the actions of others.

The aim of ethnomethodological research in mathematics education is then to understand how teachers and students interpret, construct and orient to these norms that are usually seen but unnoticed (Garfinkel 1967), as is apparent when you consider that in any interaction, 'much that is being talked about is not mentioned' (p. 221). In terms of the mathematics classroom interaction this research includes 
studies focused on the norms around turn-taking (Ingram and Elliott 2016), around argumentation and explanation (Krummheuer 2000; Yackel and Cobb 1996), the handling of student difficulties (Ingram et al. 2015; Koole 2012b; Koole and Elbers 2014), as well as teacher and student knowledge (Barwell 2003, 2013).

\subsection{Indexicality}

Indexicality refers to the context-boundedness of all interactions, what teachers and students say is reflexively related to the context in which they say it. Consequently, interactions cannot be analysed independently of the contexts in which they are generated and maintained (Heritage 1984, p. 6). However, these contexts do not just include the macro level contexts most frequently considered by mathematics education research, such as a multilingual classroom or a workplace, but also the very local context of the interaction itself which includes not only the participants involved but also what occurs before and after the interaction, or before and after an individual turn within an interaction.

Furthermore, ethnomethodologists only invoke contextual features in their analysis when it is evident from the interaction that the participants themselves are orienting to such features. In teaching and learning conversations it is often clear from the interactions themselves who is the teacher and who are the students through the structural differences in contrast to ordinary conversations. For example, teachers usually talk more, evaluate students' turns and control the topic of the conversation. Other features such as the gender or race of the participants, however, are often not so clear within an interaction. So whether a student is bilingual or from a different cultural background from others in the interaction is only relevant if it is apparent within the interaction itself. This might be through the content of the interaction such as through code-switching, but may also be apparent in the structure of the interaction such as through who generally asks the questions and who generally answers them.

This relationship with context includes reflexivity in that all social actions are context-shaped and context-renewing (Seedhouse 2004, p. 14). They can only be adequately understood by reference to the interactional context in which they occur, but they will also contribute to the immediate context for actions that follow. Through interactions teachers and students co-construct the interactional context of the mathematics classroom. Similarly, the same set of methods are responsible for both the design and construction of actions and their interpretation. Garfinkel referred to this as the 'symmetry' proposal, and the aim of ethnomethodological research is to identify these sets of methods. Researchers can then work with how utterances are designed to be recognisable within the interaction by participants as particular actions, such as requesting, eliciting, or assessing (Walsh 2013), which Schegloff (2007) describes as the 'action-formation' problem. This can lead to differences in how objects are defined. For example, from an ethnomethodological perspective an explanation is something that is treated by the participants as something that is doing the job of explaining (Ingram et al. 2016) and not a linguistic structure that meets certain criteria such as including a particular conjunction or causal inference.

The importance of local context in the analysis of interactions results in the emphasis placed on the collection of naturally occurring data. That is data collected is from 'everyday classroom practices' (Krummheuer 2011), rather than from interviews with students, experimental designed studies, or from intervention studies. In pedagogical interactions, the teacher pursues pedagogical goals, and this leads to a reflexive relationship between such goals and the "shape" of the interaction (Seedhouse 2004). In experimental or intervention studies, it is the researcher's goals that 'shape' the interaction. The data collected needs to be not constrained by a specific research design or by reference to a particular theory or conjecture. Instead, the analytic process is inductive and data driven and is described in more detail below.

\subsection{No detail can be considered irrelevant}

The final principle is that no detail can be considered 'a priori' irrelevant in the analysis. By doing so the researcher is imposing their own theories about what is relevant (Wood 2013). This requires researchers to work with video or audio recordings, or very detailed transcriptions, of classroom interactions to minimize the data that is lost through the collection methods. Field notes or coding schedules involve the observer making decisions about what to record and are therefore avoided. Similarly coding of data is not used as it reduces and simplifies actions to broad codes, and data is lost. It also influences the development of the research focus and the inductive nature of an ethnomethodological approach. A predetermined research question would focus the attention of the researcher on some aspects of the interaction whilst necessarily ignoring others, so again the analysis would be driven by the researcher's goals or theories, rather than the participants' understanding of the situation. This is not the same as not having a clear focus for the research or the analysis, but whilst the focus may be on particular phrases or pauses, the analysis should not, as far as possible, treat other features of the interaction as irrelevant during this analysis.

\section{Methods}

As mentioned above, all ethnomethodological approaches require the collection and analysis of naturally occurring data. In most studies within sociology and social 
psychology using an ethnomethodological approach, it is the participants themselves that collect the data, such as recording their mealtimes with their families or study tutorials with friends. Within mathematics education it is still usually the researcher recording the interactions (such as in the examples offered below), but as the videoing of lessons is becoming more common in schools, more recent work has involved teachers themselves videoing lessons of their choice and sharing these videos with researchers (e.g. Ingram et al. 2018). The emphasis on analysing the participants' understanding of the situation is not studied by interviewing them and asking them for their perspectives but instead from observing how they act and respond during the interaction itself. Ecological validity can be threatened if these actions differ as a result of being recorded for a specific purpose. Similarly, if we wish to study particular aspects of student or teacher talk, such as explaining, this is not done by designing interventions or specific tasks with the aim of prompting participants to explain. Instead cases of explanations that have arisen within a broader collection of naturally occurring data are the focus for an ethnomethodologically informed study. Finally, examining students' explaining practices in an experimental setting will not tell us much about explaining practices in a classroom as the nature and number of participants are different, and importantly the goals of the participants within the interaction. Interviews tell us something about students' perspectives on explaining practices (though from a CA perspective what they tell us is about is the discursive practices students use to talk about explaining), and experimental settings will tell us something about students' explaining practices in an experimental setting, but not necessarily about the actual practices within the classroom, a far more complex context.

Data is collected through audio and video recordings which are then carefully transcribed in detail on the basis that no aspect of the interaction can be considered irrelevant to the analysis. Early research using ethnomethodological approaches relied solely on the transcriptions of spoken interaction, but more recently many researchers are incorporating gestural and eye gaze information. The transcriptions usually include features such as the speed, pitch and intonation of the speech as well as the length of pauses. These detailed transcripts are rarely seen in published papers within mathematics education, however, (though see Forrester and Pike 1998 for an exception) as the level of detail or the specific use of notation can make them unreadable to a wider audience.

The analysis itself is inductive and whilst the focus of the research may be decided before the collection of data, the research questions develop through consideration of the data itself. The analysis of the data is not necessarily driven by conjectures or hypotheses, but rather evolves as the researcher notices particular features of the interaction and examines these features across cases to identify a possible underlying order within the interaction. For example, work looking at teachers' use of the word 'no' when evaluating students' responses to questions arose from noticing an avoidance of negative evaluations. This then lead to an analysis of all the cases where a teacher evaluates a students' response, including the few occasions where 'no' does occur, to identify structural differences across cases between how teachers positively and negatively evaluate a student response (Ingram et al. 2015; Seedhouse 1997). Gellert (2008) argues that these approaches result in findings having high validity, which is often further reinforced through the inclusion of the transcripts of the data being considered being shared with the reader to enable them to see where any claims have come from. However, the focus on the indexical nature of interaction means that findings cannot be generalised beyond the contexts being considered in the analysis, the level at which contexts are considered often means that you can generalise at particular levels, such as for example the structure of turn taking across classrooms (Ingram and Elliott 2014; Mchoul 1978).

\section{The contribution of ethnomethodological approaches}

In this section, I consider three key examples of the use of ethnomethodological approaches within mathematics education. One example of conversation analysis, one from Interactionism and one from discursive psychology to illustrate how the ethnomethodological approach has influenced the research design, the analysis and the conceptualization of key ideas within mathematics education. These examples serve to illustrate the focus on actions and practices such as estimating or knowing, rather than students' understanding of estimation or teachers' knowledge. Each study collected naturally occurring interactions within mathematics classrooms and the analysis focused on detailed transcriptions of these interactions.

\subsection{Conversation analysis}

Conversation analysis (CA) is distinct from discourse analysis in that the analysis of talk is underpinned by the theoretical principles of ethnomethodology. There is some disagreement within users of CA as to whether it is a type of discourse analysis or a completely discrete methodology as it focuses on actions rather than the content of talk itself. CA is now widely used in applied linguistics, medical research and legal research and there have been numerous studies that have led to changes in practice within these contexts. For example, analysis of how patients with particular medical conditions construct their identities as a person with $\mathrm{X}$ 
has altered how treatment and support is offered by doctors. Studies of how epistemics, issues of knowledge and knowing, are managed in police-suspect interviews have led to changes in how police are trained to interview suspects. Analysis of phone calls to suicide helplines have led to the identification of particular structures and features of the interaction that precede particular outcomes for the caller (see Sidnell and Stivers 2012 for some examples of these applications).

The first example within mathematics education comes from Forrester and Pike's study of what it means to estimate in a primary classroom. Through the careful analysis of the transcript using ethnomethodologically informed conversation analysis, they examine how two teachers and two groups of students within their classrooms themselves make sense of what it means to estimate in relation to measurement and show how estimation "is understood as discursively interdependent with measurement and is associated both with teacher-formulated accountability and with vagueness, ambiguity" (1998, p. 334). Their approach brings together understanding estimation both as an activity co-constructed in the classroom and estimation as a mathematical object.

Forrester and Pike particularly drew upon the temporal nature of the interactions fundamental to a $\mathrm{CA}$ approach, to show how estimation and measurement become established as distinct discursive objects and how this changed across a lesson. This was specifically through an analysis of how one teacher introduces the activities of estimating and measuring, looking specifically at the prosody of the talk, the descriptions of activities, including the construction of estimation preceding measurement, and how what it means to estimate became embedded within activity. This analysis also shows how the teacher separates measurement from estimation by associating measurement with notions of correctness, precision and using a ruler whilst associating estimation with thinking roughly, vagueness and not using a ruler. Through their subsequent analysis of small groups of students working on estimation tasks, they showed how this distinction was not as clear for the students.

Estimation is treated by the students as rough measurement which includes using non-standard measures, but not a ruler which they treat as actual measurement that temporally follows rough measurement. They further showed how the students oriented to the estimation task, the teacher (who was the one that could judge whether something was a good estimate or not) and the authority of the ruler as a means for discovering the 'real' answer, but did not orientate to the idea of estimation as a concept itself. Their analysis draws specifically on the consideration of sequence organisation combined with prosodic features of the discourse, such as what is stressed and emphasised in speech and how this changes over time.
In their paper, the authors specifically contrast their analysis to an analysis that would usually be undertaken to illustrate how these findings differ as a consequence of paying attention to what the children themselves are doing in the interaction, rather than by focusing on what evidence there is in the interaction that the children have a particular understanding of estimation as theorised in educational psychology research. Yet the analysis focuses on just two teachers and two small groups of students and there are no claims but also no sense that these specific findings might apply to the learning of estimation in general, even in similar contexts. We know from the findings that one of the teachers treated estimation and measurement as distinct discursive objects but that the small group of students from this class subsequently had a different construction of estimation from that introduced by the teacher, but we do not know if these differences were a consequence of how the teacher introduced the tasks and activities, or a consequence of the students' experiences of working on estimation tasks and activities. Similarly, to other CA research, this study gives us an in-depth insight to how what it means to estimate was constructed in these two classrooms, but not necessarily the consequences of this construction.

The second example considers the work of Krummheuer et al. (e.g. 1995, 2011, 2007) who have used Interactionism to examine argumentation in students' interactions. The ethnomethodological theoretical principles are clear in the conceptualisation of argumentation itself "as a social phenomenon, when cooperating individuals adjust their intentions and interpretations by verbally presenting the rationale of their actions" (Krummheuer 1995, p. 229). The approach focuses on the emergence of meaning within the interaction and this is revealed through the detailed examination of how meaning and relevance are negotiated by the students in the interaction. Krummheuer's work on argumentation is one clear example of where the analysis of interaction engages strongly with the mathematical aspects of the interaction. For example, he reveals a distinction between an argument being supported by the 'logic of the interaction' rather than 'the logic of the mathematical content' (2007, p. 78).

This type of analysis enables us to see the process of learning, or the development of argumentation. Krummheuer's distinctions between the nature of participation in argumentation enable a distinction between those that are learning something new to them, and those who have already learnt. Another example is Yackel and Cobb's (1996) sociomathematical norms, a concept that arose from an ethnomethodological approach to the analysis of interaction that is now widely used by mathematics education researchers using a variety of theoretical approaches (e.g. Planas and Gorgorió 2004). In each of these studies the focus is considered to be continuously and locally emerging, to 
be co-constructed by all participants through their actions in interaction.

\subsection{Discursive psychology}

Within the usual domain of psychology there are some key mental processes and constructs that are of interest to both teachers and researchers. In mathematics classrooms these processes include learning, understanding and thinking, as well as students' attitudes, identities and motivations. Discursive psychology (DP) focuses on the methods through which participants deal with these mental processes in their interactions. In particular, how psychological matters, such as knowledge and attitudes, are introduced, defined, and made relevant (Edwards and Potter 1992) in interaction. However, this approach to the analysis of the processes of psychology has not been widely taken up by researchers in mathematics education, or even educational research in general. So far in mathematics education research using this approach has focused on how knowing is made relevant (for example the work of Barwell 2003, 2013, discussed below). Whilst DP uses the same methodological approach as CA, CA examines how issues such as knowing and understanding are treated in interactions (e.g. Koole 2012a) rather than the epistemological nature of that knowledge or understanding that is the focus of DP studies (e.g. Barwell 2003, 2013). The DP approach to analysing knowledge or knowing in this way resonates with Krummheuer's (2007) and Bauersfeld (1995) and colleagues' work on knowing or ways of knowing or meaning, where knowledge is action, considered fluid and dynamic and meanings are negotiated rather than known (see also Reis and Barwell 2013).

Discursive psychology has been used by Barwell to explore the nature of mathematics knowledge in the classroom, considering both the knowledge of students (2003) and of teachers (2013). Using DP, he examines how teachers' and students' knowing is displayed in interaction, specifically through how assessments and evaluations are made, and accounts are given. Again, the ethnomethodological roots of the research are exemplified through the conceptualisation of knowledge as an action that is co-constructed by the teacher and the students in interaction. The analysis is focused on how knowing is dealt with within interactions between teachers and students, rather than on what the teacher or students know. As Barwell highlights, this approach is epistemological rather than ontological, in that the focus is the nature of knowledge as it is negotiated through interaction, with no claims about what each participant does or does not know in their heads.

This approach to considering what it means to know is dynamic and fluid as it constructed in interaction and can change as the interaction develops. The acts of knowing something, or in Barwell's (2003) paper, remembering something, are "reconceived as a discursive activity" (p. 204). However, this also means that knowing is situated within the context in which it is constructed but is also not stable or structured in ways that we often theorise it as (Edwards 1993). Thus any DP (or CA) study of mental process such as knowing or understanding focus on when and how knowing or understanding is relevant to teachers and students as they interact in the classroom, not on what they know or understand.

Each of these studies has taken a key concept that exists in the mathematics education literature and redefined it by focusing on the actions of the teachers and students themselves and how they are treating the concept in focus. This approach is applied to mathematical concepts such as estimation, mathematical practices such as argumentation, and to one of the key foci for all mathematics education research, knowledge. Each of these studies illustrates the potential contribution an ethnomethodologically informed analysis can make to the field, but these approaches are not without their critiques and challenges.

\subsection{Challenges and opportunities for ethnomethodological approaches}

Mathematics classrooms are complex social contexts in which the teaching and learning of mathematics is largely situated. Discourse within mathematics classrooms can be conceptualised as a mediational tool through which the teaching and learning of mathematics, school mathematics or other mathematical cultures takes place. This has largely been the conceptualisation used by researchers drawing upon a socio-cultural approach, but this often (necessarily) simplifies the complexity of how this discourse is interpreted by teachers and students. An ethnomethodological approach focuses on revealing this complexity and considers the role of discourse in the co-construction of what it means to teach or learn mathematics. An ethnomethodological study identifies and describes structures and patterns of interaction, which are thus opened up as opportunities for specific and targeted changes to this structure of classroom interaction (Krummheuer 2007).

One of the key challenges that researchers face is the time-consuming nature of the data collection, data analysis, and the transcription of this data in the level of detail required. This is a very practical issue that researchers also face when wanting to disseminate their work further as the transcripts need to be readable by a wider audience, but also the restrictions of space and styles in many conference proceedings and journal articles can make it difficult to share transcripts in such a way as to enable the reader to reach the same conclusions as the analyst. Furthermore, the inductive nature of the analysis leads to a risk of topic digression and multiple levels of analysis (though see 
below for examples of how this issue of multiple levels of analysis is being addressed in other fields). Forrester and Pike also warn of being "overwhelmed by the number of pre-theoretical assumptions we suspect inform the teaching practice of mathematics education" (1998, p. 354).

One critique of research using ethnomethodological approaches, but also a challenge facing researchers using these approaches, is that the research is not always clearly relevant, or clearly focused on the mathematical aspects of the interactional context. This is partly due to the fact that the focus is on describing classroom practice as it is, and not on any modification of this practice (Gellert 2008). Ethnomethodological approaches are excellent at describing how the mathematics classroom arises from the situated actions of those interacting in the classroom. However, it does not provide a way of "rethinking and remaking the world" (Roth 2005, p. 195). As a micro-level approach, the focus is on the actual doing of mathematical practices and producing of mathematical objects (Roth and Gardner 2012), it does not generally consider political or moral issues at a more macro level. It is also partly due to the inductive nature of the analysis that might result in detailed descriptions of norms, but which do not necessarily reveal how these norms affect the teaching or learning of mathematics. Ethnomethodological approaches do offer a way to understand the processes of learning, that is how students make sense of and understand a particular topic.

\section{Potential opportunities for mathematics education}

Interactionist and ethnomethodological approaches have made a significant contribution to our understanding of interaction within the mathematics classroom. Yet their influence, focus and prevalence within other fields offers us an opportunity to develop this contribution further. I will focus on two particular areas where this work could be developed further: firstly, by looking at particular topics and research questions that have been addressed in other fields using these approaches; and secondly, through examining how these approaches can be combined with other theoretical perspectives to address a wider range of research questions. The majority of these suggestions are taken from research using conversation analysis or discursive psychology and the potential opportunities taken from their use in medicine, sociology, and language education. So far within mathematics education conversation analysis and discursive psychology studies are rare in comparison to other fields such as language education, medical research and legal research.

\subsection{Potential areas for further research}

There has been considerable interest in students' identities, motivations and attitudes within mathematics education, but this has largely been studied through interviewing students. Discursive Psychology offers an approach to the study of identities, motivations and attitudes which emphasises the dynamic co-construction of these aspects through contextbound interaction. In other words, it is an ideal approach to analysing how the identity of mathematician develops and is negotiated moment-by-moment in interaction. There are considerable similarities between a Discursive Psychology approach to the analysis of data involving bilingual learners and many sociocultural approaches (Moschkovich 2002; Planas and Setati-Phakeng 2009) but these studies often only go as far as to identify differences and similarities in mathematical practices, and do not go a stage further to analyse how these differences are co-constructed in interaction.

One of the largest applications of discursive psychology in other fields is focused on identity. From an ethnomethodological perspective identity is understood to be a contextbound accomplishment of interaction. The relevance of any identity is not assumed ahead of any analysis (as being a priori relevant) but instead arises from what is consequential for speakers (Schegloff 1997). Each participant will bring with them a range of identities, for example teacher, female, sister, daughter, not all of which will be relevant or consequential to the interaction. Heritage suggests that "context and identity have to be treated as inherently locally produced, incrementally developed, and, by extension, as transformable at any moment" (Heritage 2005). The focus is on identity work which is contingent upon the context. However, in contrast to other dynamic approaches to studying identity, an ethnomethodological approach does not hold a particular view on the ontological status of identity, instead they focus on how identity positions are produced in interaction. This is similar to the approach of positioning theorists (Herbel-Eisenmann et al. 2015), where identities are co-constructed in interaction but where positioning theory also takes into account macro features of the context of the interaction which make particular identities available e.g. the context of the mathematics classroom makes the identities of teacher, student, expert, novice, learner etc. available. An ethnomethodological analysis only focuses on the micro features of the context and the aspects of identity that the participants themselves orient to in the interaction.

In other fields there are a wide range of studies that are potentially relevant to mathematics education. For example, Heritage and Raymond (2005) have done detailed work looking at the negotiation of epistemic authority within questioning in everyday conversations and showed that how a polar question (for example a question that invites a yes or no answer) is answered affects the balance of agency 
between the questioner and answerer. For example, a repetition of the content of the question shifts the balance of epistemic authority more towards the answerer than a simple yes or no response where the questioner has greater epistemic authority. Epistemics is a key focus for mathematics education and as Jungwirth argues "students can successfully participate without an understanding to be located in their 'heads'; for instance, by answering on questions by short, tentative utterances, which seem to indicate understanding so that the teacher completes to the desired answer" (in Prediger 2008, p. 282).

There has also been significant research looking at the use of particular words and how these can influence participants' responses. For example, Heritage (2010) found that "would you like some dessert" is more likely to receive an affirmative response than "would you like any dessert". Similarly, he then went on to find that doctors were more likely to get a no answer when they asked "is there anything else you want to address in the visit today" compared to when asked "is there something else you want to address in the visit today?". This raises questions for us as education researchers such as is there a difference between asking a student would you like some help and would you like any help?

Recent studies have begun to examine the relationship between social categories and classroom interaction. These often focus on the use by participants of particular social categories in the interaction (Wood 2013). These studies have paid attention to the explicit indicators in the content of the interaction, rather than any differences in the interactional practices of different groups. Many explicit references are often unavoidable in interactions through the use of gender specific pronouns for example, or pronouns used to refer to teachers such as sir or miss, but other categories are not so easily observed in talk, such as racial categories. Any teacher or student occupies many distinct and potentially relevant identity categories simultaneously such as categories of race, gender, class and sexuality as well as status related identities such as sister, daughter and so on. The challenge is then how, as researchers, we can know which categories are relevant to the interaction. One example of how CA can be combined with a quantitative approach to address this challenge is offered below (Stivers and Majid 2007).

Another issue that ethnomethodologically inclined researchers face is the need for research to have practical applications. There is a strong preference for research which leads to changes in practice and improvements in students' outcomes within mathematics. Jungwirth argues that one way to use findings from conversation analysis and Interactionist studies is to develop professional development materials that can make teachers aware of the patterns and routines within classroom interactions in order to consider alternatives (in Prediger 2008, p. 282), as has been done in other fields. As Krummheuer (2007) notes ethnomethodological studies identify the structures and patterns of interaction that exist within mathematics classroom. These patterns are relatively stable across a range of classrooms and are largely unaffected by changes to recommendations around both what to teach and how to teach it which Krummheuer uses to explain the difficulties in implementing reform approaches to teaching. However, these structures open up opportunities for targeted interventions which focus on altering these patterns of interaction so that different norms or structures can be established (e.g. Ingram and Elliott 2016).

\subsection{Combining approaches}

In this section I will outline some approaches that have combined other theoretical perspectives with an ethnomethodological one in other fields that might make a potential contribution to mathematics education. For example, whilst ethnomethodological approaches do not use coding because it reduces and flattens complex actions into simplistic codes, a range of researchers have recently begun using a mixed methods approach combining ethnomethodological approaches with quantitative methods. This enables ethnomethodological research to play a role in a wider range of research questions that they have traditionally not considered, and which may appeal to a broader audience. The challenge is to ensure the formal coding process involved in quantitative approaches does not contradict or distract from the underlying theoretical principles of an ethnomethodological approach.

One possibility would be to use quantitative approaches to examine the frequency or distribution of particular features of talk. For example, corpus linguistic methods would allow us to look at the frequency of particular pronouns, lexical bundles or mathematical vocabulary. Alternatively, coding frameworks could allow us to look at the frequency and distribution of particular structures within interactions. Conversation analysis could then be used to examine these cases of use to identify how they are being used and what is being achieved through their use by particular participants. The quantitative methods tells us 'that' something is being used whereas the ethnomethodological approach tells us 'how' it is being used.

Distributional evidence from quantitative methods is important for an argument that there is a particular norm or that there is a preference for certain activities, but it is insufficient without the participants' orientations to the practice and an understanding of the deviant cases that do not follow the generally observed pattern. The analysis of deviant cases is a key method of an ethnomethodological approach because of the further insight these offer into the norms or rules that have been identified. It is often the analysis of where something goes wrong in interaction that offers insight into the structures that were being deviated from. 
Although the combination of quantitative methods and ethnomethodological approaches has clear potential within mathematics education, the development of a coding scheme necessarily fixes the analytic frame a priori, undermining the third principle, that no detail can be considered irrelevant a priori, outlined above. One way to tackle this is to use the two methods in an iterative way, beginning with an ethnomethodological data driven analysis to identify the research focus before then using quantitative methods to narrow down this focus further and to find cases for further analysis using an Ethnomethodological approach. This was the approach taken by Walsh et al. (2011) who used corpus linguistics to identify the prevalence of functional patterns involving key words and lexical bundles within small group tutorials or seminars at university and how these patterns were used to mark particular interactional contexts. Conversation analysis was then used to analyse the contexts where these key words and lexical bundles clustered to show how they were used by the participants and to further identify four structures which marked the nature of the content of the interactions. These included structures such as procedural talk involving informing, or being informed, about procedures that were marked by phrases such as "I want you to". Two of the structures identified how the participants used the key words or lexical bundles to create a "shared space" by either building upon students' contributions or through developing arguments. The conversation analysis of these structures reveals differences in the turn taking structure, student agency and pedagogic functions. The corpus linguistics analysis revealed what linguistic devices lecturers and students were frequently using, and the conversation analysis revealed how these linguistic devises were being used to achieve different pedagogic activities.

Another approach is to use quantitative methods after the ethnomethodological analysis has been completed. This would allow research questions around whether particular interactional practices are associated with certain variables including attitudinal variables; sociodemographic variables such as age, gender, race, language, or culture; or some outcome variables such as mathematics attainment or participation in further mathematics study. Taking an example from medical research, one study combining coding and conversation analysis to examine doctor-parent-child interactions found that race was associated with next-speaker selection. Doctors were less likely to select African American children to answer questions than White or Asian children. Similarly, they also directed fewer questions to the parents rather than the children when the fathers were present in the interaction (Stivers and Majid 2007). These results show how race and gender can be socially constructed in interactions through how competence is attributed to different participants.

Fields where these approaches have been combined have been made possible through the construction of large corpora of spoken interaction. The formation of these corpora is a relatively new phenomenon within mathematics education and few of the corpora that do exist are open access and include sufficient transcription detail to be analysed using an ethnomethodological approach. Changes in technology, large collaborative projects and the drive to make data open access are leading to the emergence of some corpora but navigating the practical and ethical considerations for constructing these corpora is complex.

\section{Conclusion}

In this article I have outlined the underlying theoretical principles behind any ethnomethodological approach to research and illustrated the implications of these principles on how we conduct research. The adoption of an ethnomethodological approach requires a shift in the way key concepts within mathematics education are considered. For example, from a focus on what mathematical knowledge is, or what mathematical knowledge teachers need, to how mathematical knowing or the status of being knowledgeable is co-constructed and used by both teachers and students in interaction. Analysis of classroom interactions focuses on how teachers and students use language and structures of interaction to co-construct the interactional context to achieve particular things. What it means to learn mathematics, to do mathematics and to understand mathematics is co-constructed in these interactions. The micro analyses that ethnomethodological based approaches offer also open up opportunities for us to change what it means to learn mathematics by changing the often-implicit patterns of interaction that are used to construct the interactional context of the mathematics classroom.

There is always a risk in applying theories from other fields and as Niss (2007) points out, "many mathematics education researchers relate their work to explicitly invoked theories borrowed from other fields....and often do so in rather eclectic or vague ways" (p. 101). The risk arises from applying an approach without considering the theoretical underpinnings of the approach, for example conversation analysis without the ethnomethodological principles is just a form of discourse analysis and would not be recognised as conversation analysis by researchers in other fields. Yet the use of these ethnomethodological approaches are not as widespread as they are in other fields, including those with a similar education focus such as language education. My intention is to open up discussions about the potential for future research in mathematics education that can make use of the methodologies and concepts within ethnomethodological approaches in the ways that they have been used elsewhere. 
Open Access This article is distributed under the terms of the Creative Commons Attribution 4.0 International License (http://creativeco mmons.org/licenses/by/4.0/), which permits unrestricted use, distribution, and reproduction in any medium, provided you give appropriate credit to the original author(s) and the source, provide a link to the Creative Commons license, and indicate if changes were made.

\section{References}

Barwell, R. (2002). The development of a discursive psychology approach to investigate the participation of students with English as an additional language (EAL) in writing and solving arithmetic word problems with peers. Bristol: University of Bristol.

Barwell, R. (2003). Discursive psychology and mathematics education: possibilities and Challenges. ZDM-International Journal on Mathematics Education, 35(5), 201-207.

Barwell, R. (2013). Discursive psychology as an alternative perspective on mathematics teacher knowledge. ZDM-International Journal on Mathematics Education, 45(4), 595-606. https:// doi.org/10.1007/s11858-013-0508-4.

Bauersfeld, H. (1995). Language games in the mathematics classroom: their function and their effects. In P. Cobb \& H. Bauersfeld (Eds.) The emergence of mathematical meaning: interaction in classroom cultures (pp. 271-291). Hillsdale, NJ: Lawrence Erlbaum

Bruner, J. S. (1990). Acts of meaning. The Jerusalem-Harvard lectures. Cambridge: Harvard University Press.

Bruner, J. S. (1996). The culture of education. Cambridge: Harvard University Press.

Edwards, D. (1993). But what do children really think?: discourse analysis and conceptual content in children's talk. Cognition and Instruction, 11(3-4), 207-225.

Edwards, D., \& Potter, J. (1992). Discursive psychology. In M. Rapley \& A. Mchoul (Eds.), How to analyse talk in institutional settings: a casebook of methods. London: Continuum International.

Forrester, M. A., \& Pike, C. D. (1998). Learning to estimate in the mathematics classroom: a conversation-analytic approach. Journal for Research in Mathematics Education, 29(3), 334. https:// doi.org/10.2307/749793.

Garfinkel, H. (1967). Studies in ethnomethodology. Englewood Cliffs: Prentice-Hall

Gellert, U. (2008). Validity and relevance: comparing and combining two sociological perspectives on mathematics classroom practice. ZDM-International Journal on Mathematics Education, 40(2), 215-224. https://doi.org/10.1007/s11858-008-0074-3.

Herbel-Eisenmann, B. A. A., Wagner, D., Johnson, K. R. R., Suh, H., \& Figueras, H. (2015). Positioning in mathematics education: revelations on an imported theory. Educational Studies in Mathematics, 89(2), 185-204. https://doi.org/10.1007/s10649-014-9588-5.

Heritage, J. (1984). Garfinkel and Ethnomethodology. New York: Polity Press.

Heritage, J. (2005). Conversation analysis and institutional talk. In K. L. Fitch \& R. E. Sanders (Eds.), Handbook of language and social interaction (pp. 103-146). Mahwah: Lawrence Erlbaum Associates Inc.

Heritage, J. (2010). Questioning in Medicine. In A. Freed \& S. Ehrlich (Eds.), Why do you ask?: The function of questions in institutional discourse (pp. 42-68). Oxford: Oxford University Press.
Heritage, J., \& Raymond, G. (2005). The terms of agreement: indexing epistemic authority and subordination in talk-in-interaction. Social Psychology Quarterly, 68(1), 15-38. https://doi. org/10.1177/019027250506800103.

Ingram, J., Andrews, N., \& Pitt, A. (2018). Making student explanations relevant in whole class discussion. In J. N. Moschkovich, D. Wagner, A. Bose, J. Rodrigues \& M. Schütte (Eds.), Language and communication in mathematics education: international perspectives. Dordrecht: Springer.

Ingram, J., \& Elliott, V. (2014). Turn taking and "wait time" in classroom interactions. Journal of Pragmatics, 62, 1-12. https://doi. org/10.1016/j.pragma.2013.12.002.

Ingram, J., \& Elliott, V. (2016). A critical analysis of the role of wait time in classroom interactions and the effects on student and teacher interactional behaviours. Cambridge Journal of Education, 46(1), 1-17. https://doi.org/10.1080/03057 64X.2015.1009365.

Ingram, J., Pitt, A., \& Baldry, F. (2015). Handling errors as they arise in whole-class interactions. Research in Mathematics Education, 17(3), 183-197. https://doi.org/10.1080/14794 802.2015.1098562.

Koole, T., \& Elbers, E. (2014). Responsiveness in teacher explanations: A conversation analytical perspective on scaffolding. Linguistics and Education, 26, 57-69.

Koole, T. (2012a). Teacher evaluations: assessing "knowing", "understanding", and "doing.". In G. Rasmussen, C. E. Brouwer \& D. Day (Eds.), Evaluating Cognitive Competences in Interaction (pp. 43-66). Philadelphia: John Benjamins Publishing Company.

Koole, T. (2012b). The epistemics of student problems: explaining mathematics in a multi-lingual class. Journal of Pragmatics, 44(13), 1902-1916. https://doi.org/10.1016/j.pragm a.2012.08.006.

Krummheuer, G. (2000). Mathematics learning in narrative classroom cultures: studies of argumentation in primary mathematics education. For the Learning of Mathematics, 20(1), 22-32.

Krummheuer, G. (2007). Argumentation and participation in the primary mathematics classroom. Two episodes and related theoretical abductions. Journal of Mathematical Behavior, 26(1), 60-82. https://doi.org/10.1016/j.jmathb.2007.02.001.

Krummheuer, G. (2011). Representation of the notion "learning-asparticipation" in everyday situations of mathematics classes. ZDM-International Journal on Mathematics Education, 43(1), 81-90. https://doi.org/10.1007/s11858-010-0294-1.

Krummheuer, G. (1995). The Ethnography of Argumentation. In P. Cobb \& H. Bauersfeld (Eds.), The emergence of mathematical meaning. Interaction in classroom cultures (pp. 229-270). Hillsdale: Lawrence Erlbaum Associates Inc.

Mchoul, A. (1978). The organization of turns at formal talk in the classroom. Language in Society, 7(2), 183-213.

Moschkovich, J. N. (2002). A situated and sociocultural perspective on bilingual mathematics learners. Mathematical Thinking and Learning, 4(2-3), 189-212. https://doi.org/10.1207/S15327833M TL04023.

Moschkovich, J. N. N. (2016). Recommendations for research on language and learning mathematics. In J. Moschkovich, D. Wagner, A. Bose, R. Rodrigues \& M. Schütte (Eds.), 13th International Congress on mathematical Education. Hamburg: Springer.

Niss, M. (2007). The concept and role of theory in mathematics education. In C. Bergsten, B. Grevholm, H. Masoval, \& F. Ronning (Eds.), Relating practice and theory in mathematics education: Proceedings of NORMA 05 (pp. 97-110). Trondheim: Trondheim University Press.

Planas, N., \& Gorgorió, N. (2004). Are different students expected to learn norms differently in the mathematics classroom? Mathematics Education Research Journal, 16(1), 19-40. https://doi. org/10.1007/BF03217389. 
Planas, N., \& Setati-Phakeng, M. (2009). Bilingual students using their languages in the learning of mathematics a sociolinguistic approach to bilingualism. Mathematics Education Research Journal, 21(3), 36-59.

Prediger, S. (2008). How are theoretical approaches expressed in research practices? A report on an experience in comparing theoretical approaches with respect to the construction of research problems. ZDM-international Journal on Mathematics Education, 40(2), 277-286. https://doi.org/10.1007/s11858-008-0076-1.

Radford, L. (2008). Connecting theories in mathematics education: challenges and possibilities. ZDM-international Journal on Mathematics Education, 40(2), 317-327. https://doi.org/10.1007/ s11858-008-0090-3.

Reis, G., \& Barwell, R. (2013). The interactional accomplishment of not knowing in elementary school science and mathematics: Implications for classroom performance assessment practices. International Journal of Science and Mathematics Education, 11(5), 1067-1085.

Roth, W. (2005). Ethnomethodology and the r/evolution of science education. Canadian Journal of Science, Mathematics and Technology Education, 5(2), 185-198.

Roth, W., \& Gardner, R. (2012). “They're gonna explain to us what makes a cube a cube?" Geometrical properties as contingent achievement of sequentially ordered child-centered mathematics lessons. Mathematics Education Research Journal, 24, 323-346.

Schegloff, E. A. (1997). Whose text? Whose context? Discourse \& Society, 8(2), 165-187.

Schegloff, E. A. (2007). Sequence organization in interaction: a primer in conversation analysis. Cambridge: Cambridge University Press.
Seedhouse, P. (1997). Review article the case of the missing "No": the relationship between pedagogy and interaction. Language Learning, 47(3), 547-583.

Seedhouse, P. (2004). The interactional architecture of the language classroom: a conversation analysis perspective. Malden: Blackwell Pub.

Sidnell, J., \& Stivers, T. (2012). The handbook of conversation analysis. Wiley-Blackwell.

Stivers, T., \& Majid, A. (2007). Questioning children: Interactional evidence of implicit bias in medical interviews. Social Psychology Quarterly, 70(4), 424-441. https://doi.org/10.1177/0190272507 07000410

Walsh, S., Morton, T., \& O'Keeffe, A. (2011). Analysing university spoken interaction: a CL/CA approach. International Journal of Corpus Lingustics, 16(3), 325-344. https://doi.org/10.1075/ ijcl.16.3.03wal.

Walsh, S. (2013). Corpus linguistics and conversation analysis at the interface: theoretical perspectives, practical outcomes. In J. Romero-Trillo (Ed.), Yearbook of Corpus linguistics and pragmatics 2013: new domains and methodologies (pp. 37-51). Dordrecht: Springer Netherlands. https://doi. org/10.1007/978-94-007-6250-3_3.

Wood, M. B. (2013). Mathematical micro-identities: Moment-tomoment positioning and learning in a fourth-grade classroom. Journal for Research in Mathematics Education, 44(5), 775-808. https://doi.org/10.5951/jresematheduc.44.5.0775.

Yackel, E., \& Cobb, P. (1996). Sociomathematical norms, argumentation, and autonomy in mathematics. Journal for Research in Mathematics Education, 27(4), 458-477. 